\title{
Reply to: 'Lack of evidence for intergenerational inheritance of immune resistance to infections'
}

\author{
Natalie Katzmarski ${ }^{1,11}$, Jorge Domínguez-Andrés $\mathbb{D}^{2,3,11} \bowtie$, Branko Cirovic ${ }^{1,11}$, Georgios Renieris ${ }^{4}$, \\ Eleonora Ciarlo ${ }^{5}$, Didier Le Roy ${ }^{5}$, Konstantin Lepikhov ${ }^{6}$, Kathrin Kattler ${ }^{6}$, Gilles Gasparoni ${ }^{6}$, \\ Kristian Händler7, Heidi Theis ${ }^{7}$, Marc Beyer,8, Jos W. M. van der Meer ${ }^{2}$, Leo A. B. Joosten ${ }^{2,3}$, \\ Jörn Walter ${ }^{6}$, Joachim L. Schultze ${ }^{8,9}$, Thierry Roger5,11, Evangelos J. Giamarellos-Bourboulis ${ }^{4,11}$, \\ Andreas Schlitzer ${ }^{1,11}$ and Mihai G. Netea $\mathbb{1 D}^{2,3,10,11}$
}

REPLYING TO E. Kaufmann et al. Nature Immunology [Matters Arising https://doi.org/10.1038/s41590-021-01102-0] (2021)

\begin{abstract}
We read with great interest the study by Kaufmann and colleagues investigating the possibility that protection to reinfection may be transmitted intergenerationally in mice ${ }^{1}$. The authors found no such inheritance of resistance in models using BCG, $\beta$-glucan or a Candida albicans infection as potential inducers of trained immunity. This is different from our recent findings ${ }^{2}$ in which we show, based on independent experimental data from different laboratories, that a $C$. albicans infection or zymosan injection can induce intergenerational protection in three models of bacterial or fungal infections, and influences immunological responses to endotoxemia. We are very encouraged that this topic is gaining increased interest: it is indeed important that more information is becoming available regarding variations between different laboratories and conditions. The reason for the discrepancy between the findings in the two studies is unclear, and it likely involves local differences in mouse substrains, housing, diet, microbiome, infection models or other factors. These findings underscore the effect of the environment on the intergenerational inheritance of infection resistance. What these environmental factors are and how these factors are integrated with regards to intergenerational inheritance remains largely elusive at this time. One intriguing possibility that needs to be tested in future studies is whether such effects may be more robust in outbred wild mice, in which subtle environmental changes may have less strong impact.
\end{abstract}

A question raised by Kaufmann and colleagues is whether intergenerational transmission of resistance to infections, which is well documented in plants and invertebrate animals ${ }^{3,4}$, has been lost once vertebrates have acquired adaptive immunity. We argue that this is not the case based on a number of arguments. First, on theoretical grounds, the evolutionary advantage to transmit resistance to infection to the next generation should be independent of whether it is mediated by innate or adaptive immunity. Darwinian evolution through genetic variability and selection is a slow process, which cannot provide protection in the short term during a severe epidemic, while intergenerational transmission of resistance based on epigenetic processes has the potential to induce rapid protection in the next generation. Second, intergenerational transmission of traits induced by metabolic stresses or toxins in vertebrates has been demonstrated extensively, as also acknowledged by Kaufmann et al. ${ }^{1}$. However, these are not adaptive processes, and it is highly unlikely that the epigenetic mechanisms transmitting these traits have persisted during evolution to induce deleterious effects in the progeny. It is much more likely that the capacity to transmit changes in the next generation persisted in vertebrates to confer an evolutionary advantage, and infections are the most likely target. Third, transmission of immune traits to the next generation in vertebrates has been recently demonstrated in other studies. In mice, inflammation in males induces underweight progeny ${ }^{5}$, prenatal infection in females promotes tissue-specific immunity ${ }^{6}$ and sepsis-induced tolerance can be transmitted intergenerationally ${ }^{7}$. Additionally, in humans, lower overall mortality has been reported in infants whose fathers had been vaccinated with $\mathrm{BCG}^{8}$, and maternal SARS-CoV-2 infection has been associated with increased cytokine functionality and nonspecific immune imprinting in the neonates ${ }^{9}$. Finally, trained immunity has been shown to be transmitted in the newborn infants of hepatitis B virus-infected mothers ${ }^{10}$.

Altogether, we believe that these new data from independent studies strengthen the conclusion that intergenerational transmission of immune traits is an important characteristic of vertebrate physiology and a function of environmental priming. However, we also acknowledge, on the basis of the data of Kaufmann and colleagues, that these mechanisms are likely influenced by the environmental conditions in which such transmission occurs, and this should stimulate more research in this important new field of investigation.

'Quantitative Systems Biology, Life and Medical Sciences Institute, University of Bonn, Bonn, Germany. ${ }^{2}$ Department of Internal Medicine and Radboud Center for Infectious diseases, Radboud University Nijmegen Medical Centre, Nijmegen, the Netherlands. ${ }^{3}$ Radboud Institute for Molecular Life Sciences, Radboud University Medical Center, Nijmegen, the Netherlands. ${ }^{4} 4$ th Department of Internal Medicine, National and Kapodistrian University of Athens, Medical School, Athens, Greece. IInfectious Diseases Service, Department of Medicine, Lausanne University Hospital and University of Lausanne, Epalinges, Switzerland. ${ }^{6}$ Department of Genetics/Epigenetics, Saarland University, Saarbrücken, Germany. ${ }^{7}$ PRECISE, Platform for Single Cell Genomics and Epigenomics at the German Center for Neurodegenerative Diseases and the University of Bonn, Bonn, Germany. ${ }^{8}$ Molecular Immunology in Neurodegeneration, German Center for Neurodegenerative Diseases, Bonn, Germany. ${ }^{9}$ Department for Genomics \& Immunoregulation, Life and Medical Sciences Institute, University of Bonn, Bonn, Germany. ${ }^{10}$ Department for Immunology \& Metabolism, Life and Medical Sciences Institute, University of Bonn, Bonn, Germany. ${ }^{11 T h e s e}$ authors contributed equally: Natalie Katzmarski, Jorge Domínguez-Andrés, Branko Cirovic, Thierry Roger, Evangelos J. Giamarellos-Bourboulis, Andreas Schlitzer, Mihai G. Netea. $\varpi_{e}$-mail: jorge.dominguezandres@radboudumc.nl 


\section{Online content}

Any methods, additional references, Nature Research reporting summaries, source data, extended data, supplementary information, acknowledgements, peer review information; details of author contributions and competing interests; and statements of data and code availability are available at https://doi.org/10.1038/ s41590-021-01103-z.

Received: 18 November 2021; Accepted: 29 November 2021; Published online: 20 January 2022

\section{References}

1. Kaufmann, E. et al. Lack of evidence for intergenerational inheritance of immune resistance to infections. Nat. Immunol. https://doi.org/10.1038/ s41590-021-01102-0 (2021).

2. Katzmarski, N. et al. Transmission of trained immunity and heterologous resistance to infections across generations. Nat. Immunol. 22, 1382-1390 (2021).

3. Luna, E., Bruce, T. J. A., Roberts, M. R., Flors, V. \& Ton, J. Next-generation systemic acquired resistance. Plant Physiol. 158, 844-853 (2012).
4. Mondotte, J. A. et al. Evidence for long-lasting transgenerational antiviral immunity in insects. Cell Rep. 33, 108506 (2020).

5. Rokade, S. et al. Transient systemic inflammation in adult male mice results in underweight progeny. Am. J. Reprod. Immunol. 86, e13401 (2021).

6. Lim, A. I. et al. Prenatal maternal infection promotes tissue-specific immunity and inflammation in offspring. Science 373, eabf3002 (2021).

7. Bomans, K. et al. Paternal sepsis induces alterations of the sperm methylome and dampens offspring immune responses-an animal study. Clin. Epigenetics 10, 89 (2018)

8. Berendsen, M. et al. Parental Bacillus Calmette-Guérin vaccine scars decrease infant mortality in the first six weeks of life: a retrospective cohort study. EClinicalMedicine. 39, 101049 (2021).

9. Gee, S. et al. The legacy of maternal SARS-CoV-2 infection on the immunology of the neonate. Nat. Immunol. https://doi.org/10.1038/ s41590-021-01049-2 (2021).

10. Hong, M. et al. Trained immunity in newborn infants of HBV-infected mothers. Nat. Commun. 6, 6588 (2015).

Publisher's note Springer Nature remains neutral with regard to jurisdictional claims in published maps and institutional affiliations.

(c) The Author(s), under exclusive licence to Springer Nature America, Inc. 2022 


\section{Author contributions}

All authors contributed to writing and correcting of the manuscript.

\section{Competing interests}

The authors declare no competing interests.

\section{Additional information}

Correspondence and requests for materials should be addressed to Jorge Domínguez-Andrés.

Peer review information Zoltan Fehervari was the primary editor on this article and managed its editorial process and peer review in collaboration with the rest of the editorial team.

Reprints and permissions information is available at www.nature.com/reprints. 